\begin{tabular}{|l|l|c|c|}
\hline Eiszeitalter $u$. Gegenwart & 34 & $163-178$ & Hannover 1984 \\
\hline
\end{tabular}

\title{
Die Stellung der Stauchwälle von Kleve-Kranenburg im Rahmen der saalezeitlichen Gletschervorstöße am Niederrhein
}

\author{
Helmut Siebertz*
}

Glacial features, glaciomorphology, moraines, outwash plains, glacial tectonics (ice-pushed ridge), heavy minerals, size distribution, Middle Pleistocene, Saale Ice Age (Drenthe)

Rhenish Westphalian Basin (Kleve-Kranenburg area), North Rhine Westphalia TK 25: Nr. 4202

K u r zf assung: Der nördliche Niederrheinische Höhenzug zwischen Kleve-Kranenburg, Kalkar und Goch (Abb. 1, 2) bildet keine glazialmorphologische Einheit, wie dies häufig in der Literatur angenommen wird und in Profilen dargestellt ist (Abb. 8). Die sedimentpetrographischen und schwermineralogischen Ergebnisse, die Grundmoränenfunde und morphologischen Verhältnisse lassen den Schluß zu, daß der Höhenzug aus folgenden Einheiten besteht, die genetisch unabhängig voneinander zu betrachten sind: 1 . Sanderhochfläche zwischen Kleve, Louisendorf und Uedem; 2. Stauchwall von Moyland bei Kalkar im NE; 3. Stauchwälle von Kleve-Kranenburg im NW.

Die Ergebnisse zeigen, daß nicht jeder Eisvorstoß durch einen Stauchwall belegt sein muß. Am Niederrhein läßt sich für alle Stauchrücken nachweisen, daß exponierte Stauchwälle und ausgedehnte Sanderflächen einander ausschließen. Dies hat glazialmorphologisch zur Folge, daß das klassische Profil der glazialen Serie von Albrecht Penck in seiner genetischen Deutung überdacht werden muß.

\section{The Significance of the Ice-Pushed Ridges \\ of Kleve-Kranenburg during the Saale Ice Age in the Lower Rhine Area}

Abstract: The northern part of the "Niederrheinische Höhenzug" in the Kleve-Kranenburg, Kalkar, and Goch region (Fig. 1, 2) is no single glaciomorphological unit as is presented in the literature and profiles (Fig. 8). The results of sedimentary-petrographical and heavy-mineral analysis, the presence of ground moraines as well as the morphological conditions lead to the conclusion, that the Kleve region consists of the following genetically independent units: 1 . the flat upland area, i.e. the outwash plain near Kleve, Louisendorf, and Uedem; 2. the ice-pushed ridge at Moyland near Kalkar to the north-east; 3. the ice-pushed ridges at Kleve-Kranenburg to the north-west.

The findings show, that not every ice advance resulted in an ice-pushed ridge. It could be proved, that exposed ice-pushed ridges and extended outwash plains in the lower Rhine area do not exist within close range of each other. Thus glacio-morphologically, the classical profile of the glaziale Serie by Albrecht Penck must be newly considered in its genetical interpretation.

*) Anschrift des Autors: Dipl. Geogr. Dr. H. SieberTz, Seminar für Geographie, Pädagogische Fakultät, Universität Bonn, Römerstraße 164, 5300 Bonn. 


\section{Einleitung und Problemstellung}

Das Inlandeis stieß im Saale-Glazial (Drenthe-Stadial; Amersfoort-Stadium) weit nach W vor. Der Vorstoß erfolgte in einzelnen Loben, denen verschiedene Eisrandbildungen zugeordnet sind. Nach Tноме (1958) lassen sich von S nach N die Loben von Düsseldorf, Moers (Schaephuysener-Höhenzug), Xanten, Kranenburg und Valburg unterscheiden, die von KAISER (1961) durch den Lobus der Gelderschen Vallei ergänzt wurden. Die Stauchwälle von Kleve-Kranenburg sind genetisch mit dem Eislobus von Kranenburg in Verbindung zu bringen (Abb. 1).

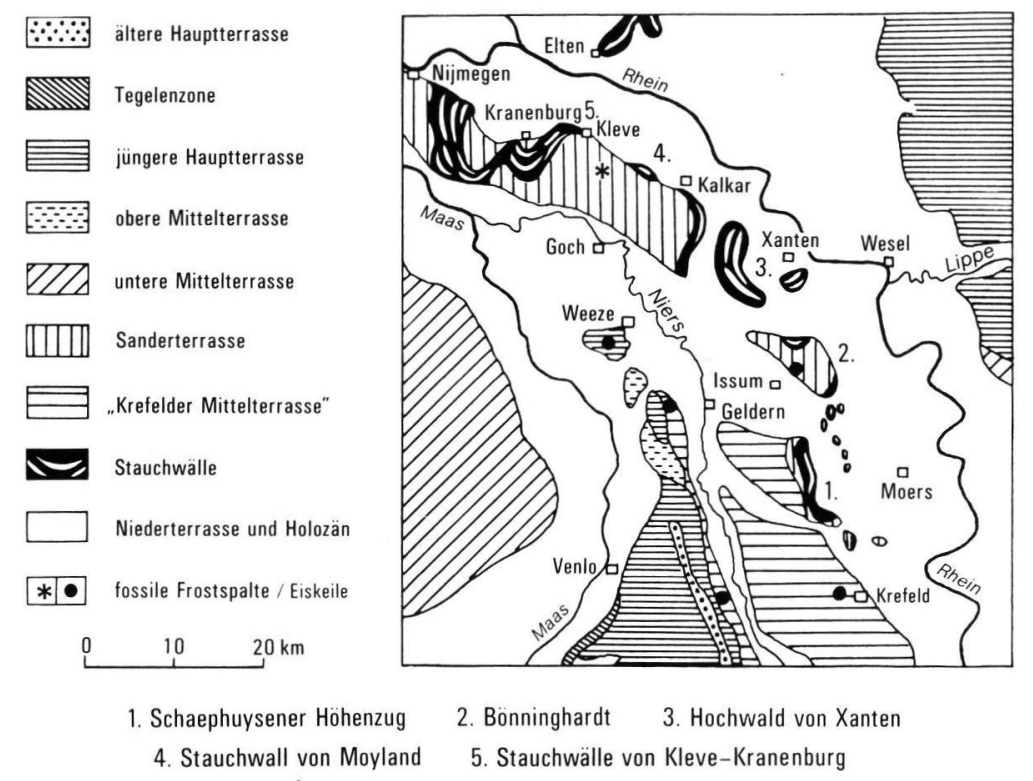

Abb. 1: Geomorphologische Skizze des Niederrheingebietes (verändert nach WAGNER 1968).

Aus den Untersuchungen von Braun (1978a), Kaiser \& Schütrumpf (1960), Heine (1983), Siebertz $(1980)$ und Thome $(1958,1959)$ geht hervor, daß das Eis am Niederrhein zweimal vorstieß. Der erste Eisvorstoß reichte am weitesten nach W, während der zweite, ein schwächerer Vorstoß, nicht mehr die Ausdehnung des ersten erreichte. Für den Raum Louisendorf-Moyland bei Kalkar (Abb. 2) konnten von SieberTz (1983) mit Hilfe eingelagerter Grundmoräne in den Sandersedimenten drei Eisvorstöße nachgewiesen werden.

Eine glazialmorphologische Schlüsselstellung auf dem nördlichen Niederrheinischen Höhenzug haben die Aufschlüsse A 12 bei Louisendorf und A 19 im Stauchwall von Moyland (Abb. 2; Beilage 1 in SieberTz 1980). Diese Aufschlüsse wurden in der Vergangenheit unter den verschiedensten glazial-geologischen und -morphologischen Aspekten untersucht (vgl. Braun 1964, 1968, 1978a; Heine 1983; Siebertz 1980, 1983).

Über die Stauchwälle von Kleve-Kranenburg ist - außer den Beschreibungen verschiedener Aufschlüsse von Braun (1968) - nicht viel bekannt. Deshalb wird mit Hilfe von sedi- 


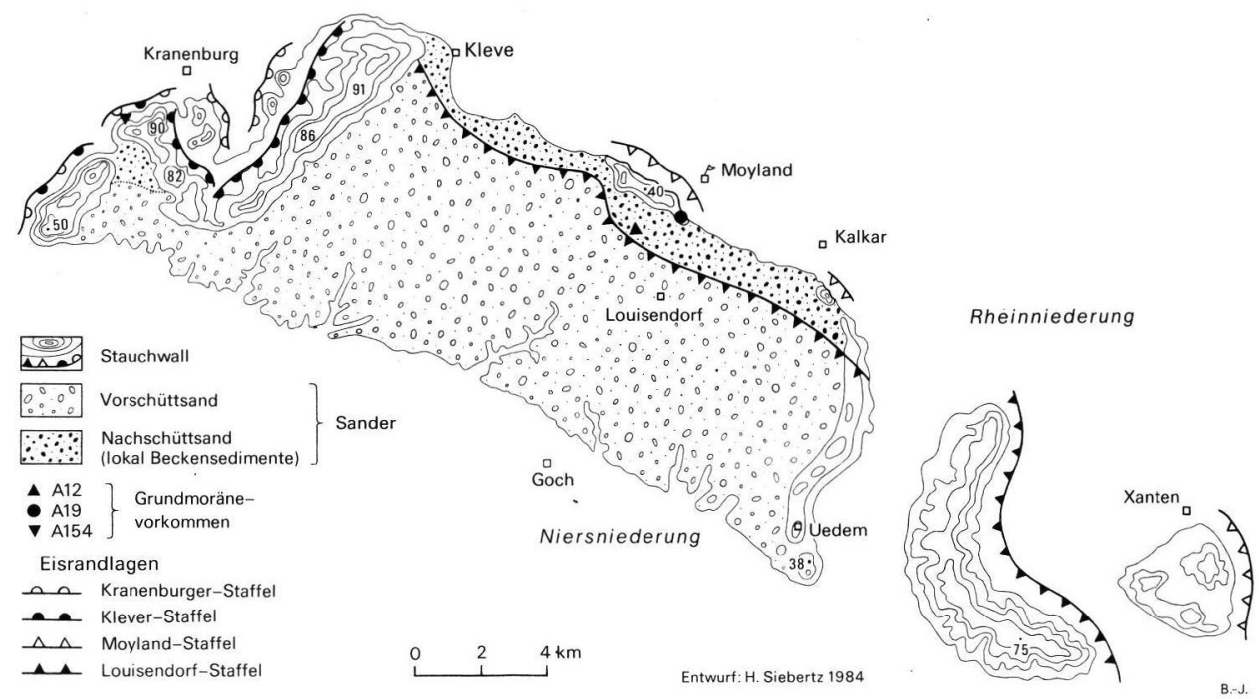

Abb. 2: Saalezeitliche Gletschervorstöße im unteren Niederrheingebiet.

mentpetrographischen Untersuchungen (Sand- und Geröllanalysen), der Verteilung von Schwermineralen in den am Aufbau beteiligten unterschiedlichen Sedimenten, der Lage von Grundmoränen sowie diverser glazialmorphologischer Befunde im Untersuchungsgebiet versucht, die Stauchwälle in das glazialmorphologische System der Eisvorstöße am Niederrhein im Saale-Glazial einzuordnen und ihren Einfluß auf die Genese dieses Raumes aufzuzeigen.

\section{Die sedimentpetrographische Zusammensetzung von Stauchwall und Sander}

Der Sander besitzt keine einheitliche sedimentologische Zusammensetzung. Deshalb wurde von SieberTz (1983) der Versuch einer Sandergliederung unternommen, die zu folgendem Ergebnis führte: die Sanderablagerungen bestehen aus vier Sedimentgruppen; 1. Vorschüttsande $\left(S_{1}\right), 2$. Nachschüttsande $\left(S_{2}\right), 3$. Beckensedimente $\left(S_{3}\right)$ und 4. $\mathrm{Bändertone} \mathrm{mit} \mathrm{ihren} \mathrm{charakteristischen} \mathrm{Korngrößenzusammensetzungen} \mathrm{(Abb.} \mathrm{3;} \mathrm{vgl.}$ Tab. 1 in Siebertz 1983) ${ }^{1}$ ).

Bei der Auswertung zeigte sich, daß für fluvioglaziale Sedimente ein Konzentrationsdreieck mit den Einteilungen Sand-Schluff-Ton nicht ausreichte, um die unterschiedlichen Sedimente abzugrenzen und in der Darstellung voll zur Geltung bringen zu können

$\left.{ }^{1}\right)$ Vorschüttsande sind gröbere Sedimentablagerungen, die beim Vorrücken des Gletschers abgelagert werden; Nachschüttsande sind charakteristisch für das Zurückweichen des Gletschers. Beckensedimente und Bändertone werden beim Eiszerfall in abflußlosen Becken gebildet. 


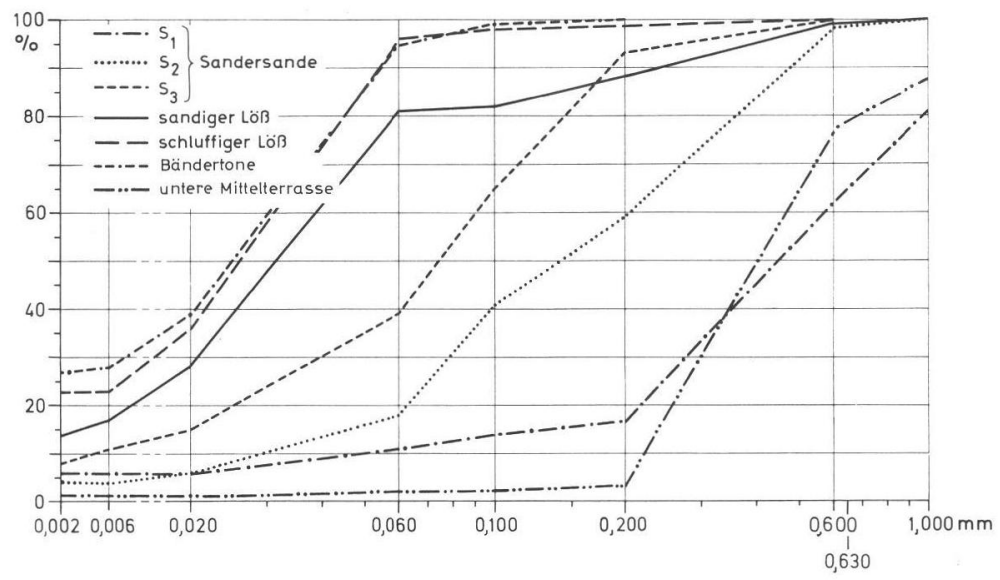

Abb. 3: Kornsummenkurven der am Aufbau des Niederrheinischen Höhenzuges beteiligten Sedimente.

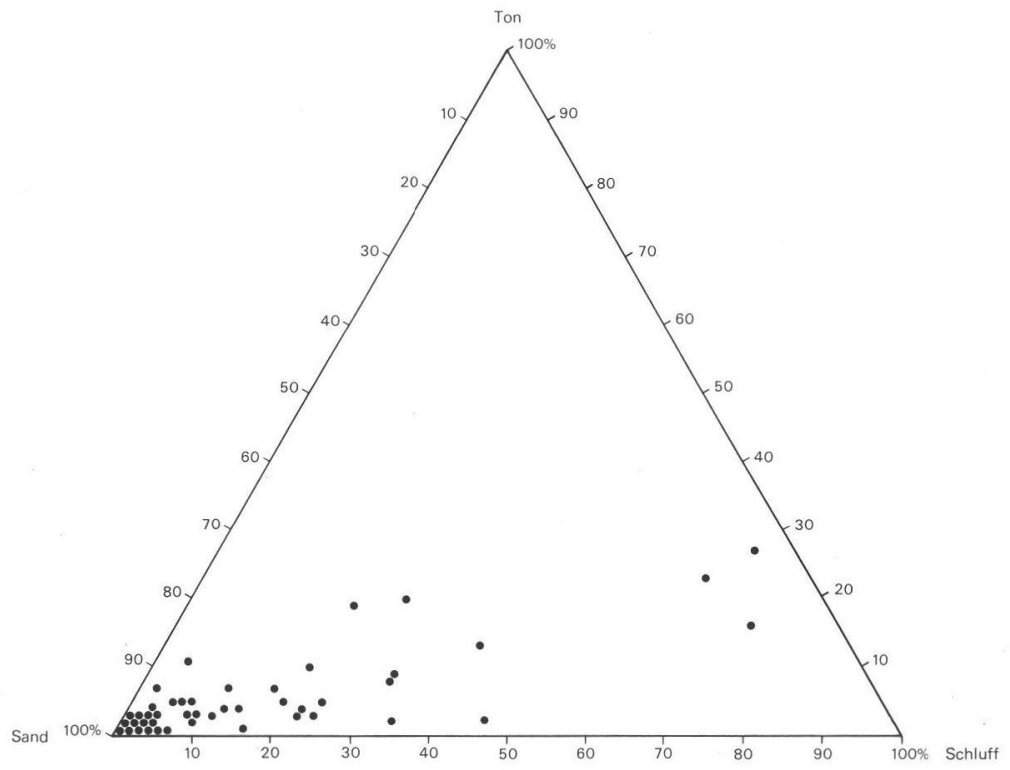

Abb. 4: Sedimentationsdreieck der am Sanderaufbau beteiligten Sedimente. 
(Abb. 4). Deshalb wurde mit Korngrößengruppen gearbeitet, die aus Grob-/Mittelsand, Feinsand, Schluff/Ton bestanden (Abb. 5) $)^{2}$ ).

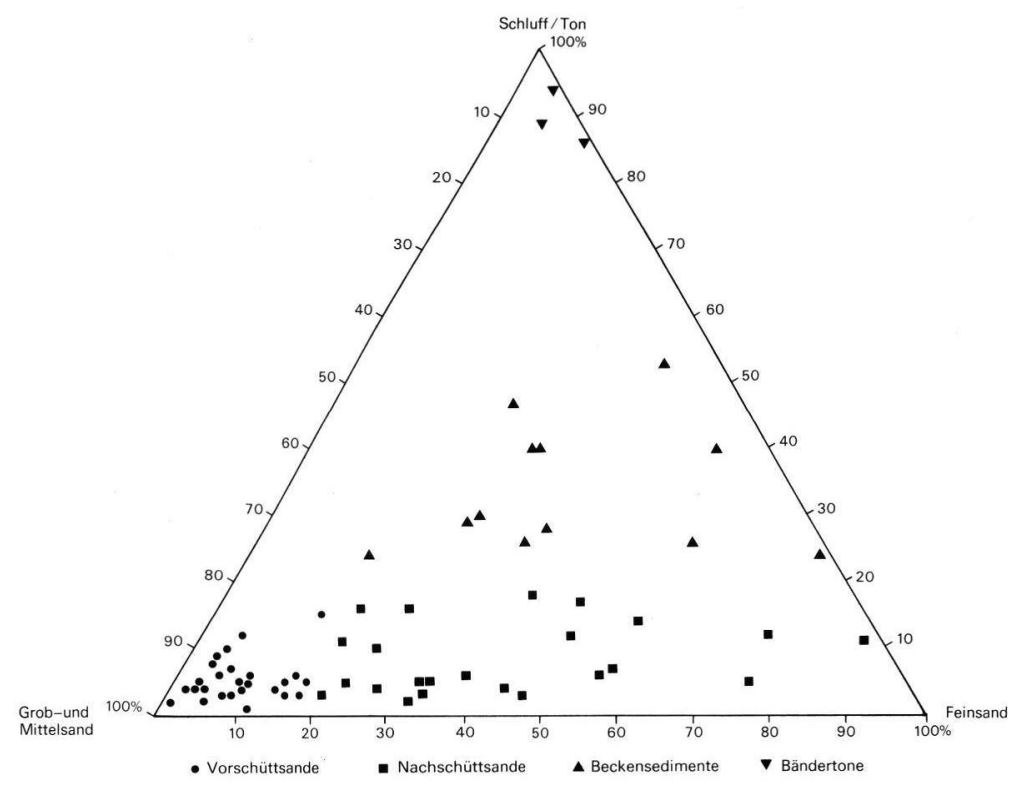

Abb. 5: Korngruppendreieck der am Sanderaufbau beteiligten Sedimente.

Die Verteilung der Sedimente in Abb. 2 zeigt deutlich, daß die Vorschüttsande die dominierenden Sandersedimente darstellen und - bis auf wenige Lokalitäten - den gesamten Höhenzug aufbauen. Nachschüttsande konnten lediglich östlich von Louisendorf in einem zusammenhängenden Areal kartiert werden (Abb. 2; Beilage 1 in SieberTz 1980). Ein geringes Vorkommen von Nachschüttsanden lagert im NW des Höhenzuges (Abb. 2).

2) Siebfraktionen $(2-0,063 \mathrm{~mm})$ mit einem Kornanteil von etwa $20 \%$ und mehr zeigen bei mehrmaliger Bearbeitung im Labor, daß sich im Vergleich verschiedener Analysen Toleranzwerte bis zu $5 \%$ einstellen können. Auf die mathematische und graphische Auswertung hat dies jedoch kaum Einfluß.

Bei den bearbeiteten Proben handelt es sich (meistens) um Repräsentativwerte, die der Charakteristik des Aufschlußprofils entsprechen. Der Aufbau eines Sedimentprofils zeigt allerdings selten eine homogene Zusammensetzung; häufig wechseln gröbere und feinere Absätze, so daß im Hinblick auf die sedimentologische Bearbeitung die Subjektivität des Bearbeiters Einfluß gewinnt. Hierbei ist es wichtig, die für das Profil zutreffende charakteristische Probe zu entnehmen.

Die Untersuchung zeigt, daß in den Vorschüttsanden das Verhältnis von gröberen zu feineren Ablagerungen etwa 2:1 ist; die Nachschüttsande weisen ein umgekehrtes Verhältnis auf. Beckensedimente sind im allgemeinen homogener und feiner aufgebaut, so daß neben geringen Grobsand- und Kiesablagerungen vornehmlich Mittel-, Feinsand und Schluffe auftreten (vgl. Tab. 1 in SiEBerTz 1983). 
Beckensedimente, wie sie aus dem Raum Louisendorf-Moyland beschrieben wurden (vgl. SIEBERTZ 1980, 1983), sind aus den Stauchwallaufschlüssen bei Kleve nur vom Wolfsberg bei Nütterden bekannt (vgl. Braun 1968).

Der Sander setzt sich vornehmlich aus fluvioglazial umgelagertem Terrassenmaterial zusammen, während die Stauchwälle nach BrAUN $(1956,1959)$ und KAISER (1961) durchweg aus aufgestauchten Kiesen und Sanden der unteren Mittelterrasse aufgebaut sind. Sie sind lokal von eingeschuppten tertiären Sedimenten durchsetzt (HeINE \& SiEBERTz 1980; KaISER 1961). Die untere Mittelterrasse zeigt eine gröbere Korngrößenzusammensetzung mit hohem Grob- und Mittelsandanteil, wie ihn die Vorschüttsande nicht aufweisen. Die Zusammensetzung einer solchen Probe vom Hey-Berg bei Frasselt ist neben denen des Sanders und dessen Decksedimente in Abb. 3 dargestellt.

Außerhalb der Stauchwälle, Grundmoränen und Steinsohlen fanden sich (meistens) keine Gerölle, deren Längsachse im Durchmesser $10 \mathrm{~cm}$ überschritt. Morphometrische Messungen an Geröllen im Hangenden verschiedener Sanderaufschlüsse $\left(D_{3}-\right.$ Steinsohle an der Lößbasis in Abb. 6) zeigen, daß die Geröllgeröße von $10 \mathrm{~cm}$ im allgemeinen nicht

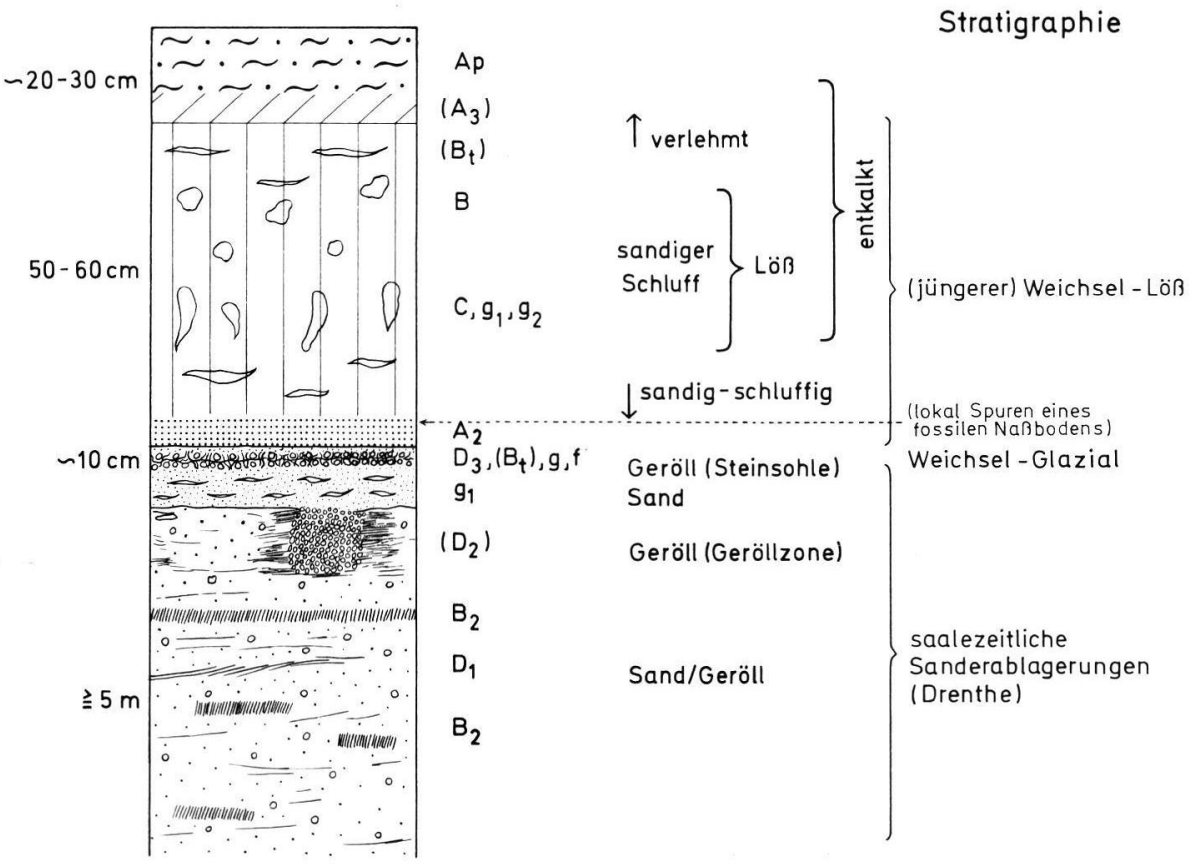

Abb. 6: Stratigraphischer Aufbau des Sanders auf dem nördlichen Niederrheinischen Höhenzug.

überschritten wird. Lediglich in den fluvioglazialen Uferwällen im Sander $\left(\mathrm{D}_{2}\right.$ in $\mathrm{Abb}$. 6) treten Gerölle bis zu $20 \mathrm{~cm}$ Kantenlänge auf (vgl. SIEBERTZ 1980). Beide Ablagerungen ( $D_{2}$ und $\mathrm{D}_{3}$ ) führen Geröllgrößen, wie sie für den Sander untypisch sind.

De Jong (1956) fand in den Stauchwällen bei Kleve einen Quarzanteil in der Fraktion $5-13 \mathrm{~mm}$ im braunen Sand bei Donsbrüggen von $66 \%$, im Hingstberg von $60 \%$ und im Wolfsberg von $66 \%$. 
Untersuchungen an Geröllen der Steinsohle (Abb. 6) im Vorschüttsand (Raum Pfalzdorf) in der Fraktion 2-10 cm ergaben folgende Werte der Quarzverteilung: A 1 R 25.12000, H 57.30700, 2-10 cm 85\%, 2-5 cm 85\%; A $2-$ R 25.13360, H 57.30680, 2-10 cm 78\%, 2-5 cm 80\%; A $3-\mathrm{R} 25.12400$, H 57.34700, 2-10 cm 67\%, 2-5 cm 72\%; A $24-$ R 25.11140, H 57.35100, 2-10 cm 70\%, 2-20 cm 70\%; A 12 (Nachschüttsand) R 25.15350, H 57.34200, 2-10 cm 70\%, 2-5 cm 73\%. Die Quarzgeröllanalyse an einem fluvioglazialen Uferwall im Aufschluß A 2 zeigte folgendes Bild: $2-20 \mathrm{~cm} 63 \%, 5-10 \mathrm{~cm}$ $58 \%$, sowie $1 \%$ nordisches Gestein (Abb. 6; vgl. MaArleveld 1956).

Aus der Steinsohle wurde bisher in keinem Falle nordisches Material nachgewiesen. Dies bedeutet nicht, daß nordische Geschiebe in der Steinsohle völlig fehlen. Dafür konnten in den fluvioglazialen Uferwällen $\left(\mathrm{D}_{2}\right)$, die zweifellos älter als die Steinsohle sind (Abb. 6), vereinzelt nordische Geschiebe nachgewiesen werden (vgl. SiEBERTz 1980).

Die Vorschütt-, aber auch die Nachschüttsande enthalten selten nordische Geschiebe; dies scheint durchaus typisch zu sein. Thiermann (1970a, b) erklärt dies mit der kräftigen Aufarbeitung des einheimischen Materials im Vorland des Gletschers, so daß bei den Vorschüttsanden der Anteil nordischer Gesteine selten über 5\% hinausgeht. Ähnliche Ergebnisse werden auch bei den Nachschüttsanden erreicht, allerdings liegt hier der Prozentsatz bei etwa $10 \%$.

Größere Mengen von nordischen Geröllen wurde lediglich im Stauchwall von Moyland (A 19 - R 25.16600, H 57.34300 in Abb. 2) während der Kiesbaggerei aus der Tiefe gefördert. Nach BRAun (1978a) führen hier die oberen Lagen der gestauchten unteren Mittelterrasse 4-8\% nordische Gerölle; die Sandfraktion enthält 8-10\% nordisches Geschiebe.

\section{Schwermineralzusammensetzungen der am glazialen Sedimentaufbau beteiligten Ablagerungen am unteren Niederrhein}

Die Schwermineralproben wurden nach Braun (1968, 1978b), KaISER (1961) und ZonNEVELD (1956) zusammengestellt (Tab. 1); sie führen zu folgender Erkenntnis: (1) Für die Schwermineralzusammensetzung der unteren Mittelterrasse ist der hohe prozentuale Augitanteil auffallend (Tab. 1). De Jong (1956) gibt den Augitgehalt für die grobkörnigen Sande im gestauchten Paket (im gefrorenen Zustand gestauchte untere Mittelterrasse) mit 70-80\% an. Das plötzliche Auftreten des hohen Augitgehaltes ist nach Zonneveld (1956) eine Folge vom Aufleben des Eifelvulkanismus. In den Sandersedimenten nimmt der Augitanteil allerdings rapide ab, wobei sich die Hornblende gegenüber dem Augit stabil verhält (vgl. Tab. 1).

VAN Andel (1952) ist der Auffassung, daß in dem von Flüssen transportierten Material keine Auflösung stattfindet; die mechanische Zerkleinerung frischer Körner sei im Flußtransport zu vernachlässigen. Dies kommt in dem hohen Augitanteil der unteren Mittelterrasse deutlich zum Ausdruck (Tab. 1). Daß dennoch in den Gletscherablagerungen die Pyroxene prozentual zurückgehen, ist sicherlich auf die mechanische Beanspruchung durch das Gletschereis, die physikalische Verwitterung und die (wahrscheinlich mehrfache) fluvioglaziale Umlagerung der Sedimente zurückzuführen.

(2) Die Schwermineralprobe des Sanders bei Goch (Tab. 1) zeigt ein Spektrum, das abgesehen von dem niedrigen Augitanteil - der unteren Mittelterrasse sehr nahe steht. Diese Sanderprobe entstammt dem Vorschüttsand, der wenig oder gar keine nordischen Geschiebe enthält (vgl. Thiermann 1970a, b). 
Tab. 1: Schwerminerale saalezeitlicher Gletscherablagerungen und tertiärer Sande am unteren Niederrhein (in \%)

\begin{tabular}{|c|c|c|c|c|c|c|c|}
\hline $\begin{array}{l}\text { Augit }+ \text { Hornblende } \\
\text { (magm.) }\end{array}$ & $\begin{array}{c}\text { ZONNEVELD } \\
(1956) \\
\text { untere } \\
\text { Mittelterrasse } \\
\text { Krefeld } \\
\\
58 \\
\text { (Augit 44) }\end{array}$ & $\begin{array}{c}\text { KAISER } \\
(1961) \\
\text { untere } \\
\text { Mittelterrasse } \\
\text { Niederrhein } \\
43 \\
\text { (Augit 31) }\end{array}$ & $\begin{array}{c}\text { KAISER } \\
(1961) \\
\text { Eisrand- } \\
\text { ablagerungen } \\
\text { Niederrhein } \\
14 \\
\text { (Augit 4) }\end{array}$ & $\begin{array}{l}\text { KAISER } \\
(1961) \\
\text { Sander } \\
\text { Vorschürt- } \\
\text { sand } \\
\text { Goch } \\
21 \\
\text { Augit 8) }\end{array}$ & $\begin{array}{c}\text { BRAUN } \\
(1968) \\
\text { Nach- } \\
\text { schüttsand } \\
\text { Aufschluß } \\
\text { A } 19 \\
10\end{array}$ & $\begin{array}{c}\text { BRAUN } \\
(1968) \\
\text { Becken- } \\
\text { sedimente } \\
\text { Aufschluß } \\
\text { A 19 } \\
2\end{array}$ & $\begin{array}{c}\text { BRAUN } \\
\text { (1978b) } \\
\text { tertiärer Sand } \\
\text { Goch- } \\
\text { fortzberg } \\
\text { Uedem } \\
46 \\
\text { Hornblende } \\
\text { (+ Augit) }\end{array}$ \\
\hline $\begin{array}{l}\text { Epidotit + Zoisit } \\
\text { (+Saussurit) }\end{array}$ & $\begin{array}{cc}18 \\
\text { (Saussurit 15) }\end{array}$ & 12 & 20 & 17 & 20 & 18 & - \\
\hline $\begin{array}{l}\text { Disthen, } \\
\text { Andalusit, } \\
\text { Sillimanit }\end{array}$ & - & 2 & 3 & 6 & 5 & 6 & 5 \\
\hline $\begin{array}{cc}\text { Turmalin } & \begin{array}{c}\text { (pegm.- } \\
\text { pneum.) }\end{array} \\
\end{array}$ & 1 & 2 & 7 & 9 & 9 & 39 & 16 \\
\hline (magm.) & 1 & 2 & 19 & 6 & 1 & 14 & 27 \\
\hline Alterit & 13 & 21 & 14 & 23 & - & - & - \\
\hline
\end{tabular}


(3) Die Eisrandablagerungen (Stauchwälle) zeigen einen erhöhten Zirkongehalt (Tab. 1). Zirkon ist in marinen Sedimenten relativ regelmäßig zu finden, so daß eingearbeitetes Tertiär - im Untergrund anstehend (Abb. 8) - zu dieser Anreicherung führte. Den Zirkon der Schmelzwassersedimente in Niedersachsen z.B. leitet HeNNINGSEN (1983) aus tertiären Ablagerungen ab.

(4) Die Nachschüttsande zeigen gegenüber der unteren Mittelterrasse einen hohen Granatanteil (BRAUN 1964, 1968, 1978a), wobei die Herkunft der Granate bisher nicht geklärt ist. Nach WeYL (1950) ist die Deutung des Granats schwierig, da er einerseits gegenüber der chemischen Verwitterung relativ empfindlich ist, andererseits aber durch die mechanische Beanspruchung des Sediments erheblich angereichert werden kann.

Tertiäre Sande des Mittelmiozäns (Braunkohlensande) und Pliozäns (s. Abb. 8) zeigen nach WEYL (1949) aufgrund der Verwitterung eine relative Anreicherung des Granats unter Verlust von Hornblende und Epidotit. Dies darf für den Nachschüttsand im Hinblick auf das Schwermineralverhältnis von Granat zu Hornblende angenommen werden (Tab. 1). Da der Nachschüttsand die untere Mittelterrasse repräsentiert, handelt es sich bei dem hohen Granatgehalt (wahrscheinlich) um tertiäre Beimengungen ${ }^{3}$ ).

(5) Die Beckensedimente zeigen gegenüber der unteren Mittelterrasse sowie den Vorund Nachschüttsanden ein völlig anderes Schwermineralspektrum (Tab. 1) mit nordischen sowie tertiären Komponenten. Letztere führen viel Turmalin, der nach DE JoNg (1956) neben Staurolith für die tertiären Sande charakteristisch ist. Daß die Granate in den Beckensedimenten schwach vertreten sind, ist darauf zurückzuführen, daß durch die Verwitterung die Anreicherung stabiler Minerale (z.B. in den tertiären Sanden) auf rund 50\% das Verschwinden des Granats verursacht (WEYL 1949).

Die schwermineralogische Zusammensetzung der tertiären Sandprobe (Tab. 1) scheint die Aussagen von WeYL (1949) über die unter bestimmten Bedingungen zu erwartenden Anreicherungen spezifischer Schwerminerale in tertiären Sedimenten zu bestätigen. In den tertiären Sanden am Niederrhein treten in der Schwermineralzusammensetzung Singularitäten auf, die zu folgenden alternativen Schlüssen führen (vgl. Tab. 1): 1. eine relative Anreicherung des Granats unter Verlust von Hornblende und Epidotit im Nachschüttsand; 2. ein hoher Turmalingehalt, der neben Staurolith für diese Sedimente charakteristisch ist im Beckensediment; 3. das Verschwinden des Granats unter relativer Anreicherung der Hornblende im tertiären Sand; 4. das Verschwinden des Granats bei einer Anreicherung stabiler Minerale auf rund 50\% im Beckensediment und tertiären Sand.

Die Beckensedimente sind reich an Feldspäten und Glimmermineralen; sie zeigen deutlich die Aufarbeitung kristalliner Geschiebe nordischer Herkunft. Im Aufschluß A 19 im Stauchwall bei Moyland (Abb.2) zeigen die Leichtminerale der Beckensedimente ein Quarz-Feldspatverhältnis von 85:15, im Gegensatz zum Nachschüttsand von 95 : 5 (BRAUN 1964, 1968, 1978a).

Die Ergebnisse zeigen, daß bei dem großen Anteil von Schwermineralen tertiärer Herkunft der Spielraum für den Nachweis nordischer Anteile sehr eng ist. Dies liegt darin begründet, daß die Anzahl der für eine sinnvolle Deutung zur Verfügung stehenden Schwerminerale begrenzt ist (vgl. Tab. 1), charakteristische Schwerminerale für nordisches Geschiebe aber auch schwer nachweisbar sind. Die in Tab. 1 aufgeführten Schwerminerale sind vom Edukt her in den meisten klastischen Sedimenten (relativ regelmäßig) zu finden (vgl. tertiäre Sande), so daß lediglich durch geologische Ereignisse charakteristische Schwer-

3) Nach Henningsen (1983) kommen die Granate in den Sandersedimenten im E und NE Niedersachsens überwiegend aus Skandinavien. 
mineralassoziationen hervorgebracht werden können (vgl. Einfluß des Eifelvulkanismus auf das Schwermineralbild der unteren Mittelterrasse). Das geringe Geschiebevorkommen am gesamten glazialen Sedimentaufbau im Niederrheingebiet - vielleicht aber auch das des metamorphen Kristallins im Hinblick auf den Granatanteil - rechtfertigt am Niederrhein daher kaum eine schwermineralogische Verbindung zu den nordischen Geschieben zu ziehen.

\section{Die Bedeutung der Grundmoräne für die Bildung der Stauchwälle von Kleve-Kranenburg}

Die Stauchwälle von Kleve-Kranenburg sind - bis auf vielleicht wenige unbekannte Lokalitäten - völlig frei von auflagernder Grundmoräne. Lediglich im Aufschluß A 154 (R 24.99590, H 57.37480) im äußersten NW des Stauchwalls bei Kranenburg (Abb. 2) wurde in einer Grube von relativ niedriger Höhe ( $40 \mathrm{~m}$ über NN) Grundmoräne gefunden.

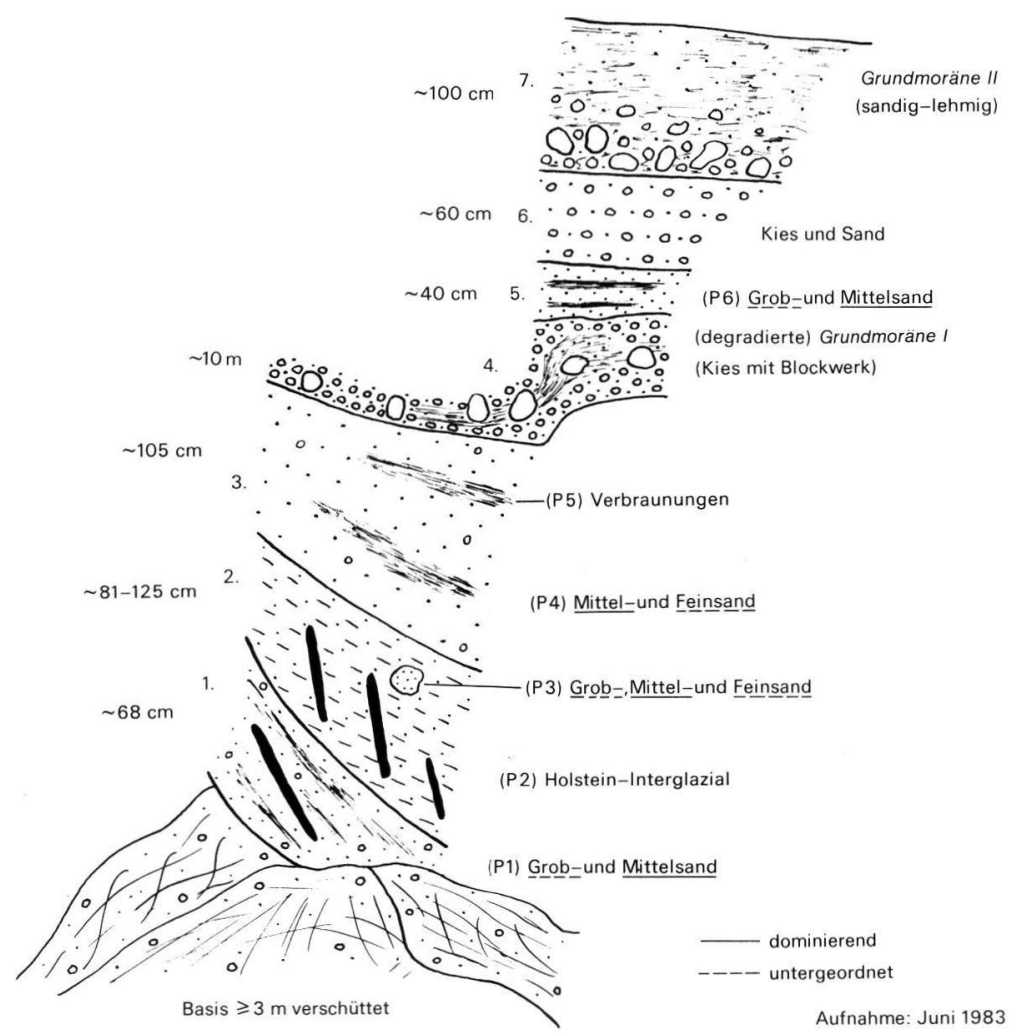

Abb. 7: Stauchwallprofil A 154 bei Kranenburg (R 24.99590; H 57.37480). 


\section{Profilbeschreibung des Aufschlusses A 154 (Abb. 7)}

1. Die Basis wird von einem gelben Grob- und Mittelsand aufgebaut, der von vereinzelten Kiesen durchsetzt ist. Stellenweise sind graublaue (tertiäre) Sande mit eingearbeitet. Dieser Abschnitt zeigt lokal kräftige Oxidationsspuren und eingestauchte schmale grau-grüne Tonstreifen, deren Aussehen den Reduktionshorizonten in der Rheinniederung ähnelt (P 1).

2. (P 2) bildet einen tonigen, schwach sandigen Abschnitt, der mit dunkelbraun-schwarzen Streifen (Holstein-Interglazial) durchsetzt ist. Lokal sind kleine hell-blaue tertiäre Sandschuppen (P 3) eingepreßt.

3. (P 4) setzt sich aus grau-blauem Mittel- und Feinsand zusammen, der lokal von kleinen Kiesen und kräftigen Oxidationszonen (P 5) durchsetzt ist.

4. Dieser Profilabschnitt bildet den Rest einer gestauchten Grundmoräne; sie wurde aufgrund der Abtragung bis auf ihre aus Kies und Blockwerk bestehende Basis degradiert.

5. (P 6) wird von einem hellen Grob- und Mittelsand aufgebaut, der von kräftigen Verbraunungsabschnitten durchsetzt ist.

6. Hierbei handelt es sich um eine von Sand durchsetzte Kiesschicht.

7. Das Hangende des Profils bildet eine dunkelbraune, sandig, schwach lehmige Grundmoräne, deren Basis aus einer kräftigen Kiesschicht mit Blockwerk gemischt aufgebaut wird.

In den Stauchwällen von Kleve-Kranenburg lassen sich zwei Grundmoränen nachweisen, die durch Kies- und'Sandschichten voneinander getrennt sind. Die Grundmoräne (I) ist bis auf die Basis, welche sich aus Kiesen und Blockwerk zusammensetzt, abgetragen worden (Abb. 7). Dies ist durchaus normal, denn auch in den Niederlanden hat man niemals mehrere und nur selten zwei Grundmoränen aus der Saale-Kaltzeit übereinander gefunden und durch fluvioglaziale Sande getrennt. Es ist durchaus möglich, daß die Grundmoräne einer älteren Vorstoßphase im allgemeinen vollständig abgetragen worden war, bevor die Grundmoräne eines folgenden Vorstoßes zur Ablagerung kam (ZANDSTRA 1976), was durchaus das geringe Vorkommen von Grundmoräne in den niederrheinischen Stauchwällen und die Abtragung der Grundmoräne (I) erklärt. Die Grundmoräne (II) im Hangenden des Aufschlusses zeigt dagegen eine charakteristische Zusammensetzung, die mit Kies und Blockwerk an der Basis beginnt und schließlich in den typischen braunen Geschiebesand übergeht.

Die Entstehung der Grundmoräne ist nach Gripp (1974) auf die Randzone des fließenden Eises beschränkt. Mit der Grundmoränenablagerung werden somit die Eisstände und Eisvorstöße dokumentiert. Von den meisten niederländischen und niederrheinischen Stauchwällen ist bekannt, daß das Gletschereis die Stauchwallhöhen nicht überschritten hat. Braun (1959) bemerkt, daß ein großer Teil der Stauchwälle einmal von einer dünnen Grundmoräne bedeckt gewesen sein muß, was aus dem vereinzelten Auftreten von nordischem Geschiebe hervorgeht. Dies kann allerdings nur für die niedrigen Stauchwälle gelten, denn Beobachtungen in den Niederlanden von DE ZANGER (1980) haben ergeben, daß unterhalb eines bestimmten Niveaus mehr nordische Gesteine vorkommen als oberhalb. Grundmoräne und Findlinge konnten auf dem Stauchwall von Arnheim noch in $70 \mathrm{~m}$ Höhe beobachtet werden (De Zanger 1980), während bei Edelman \& MaArleveld (1958) für die Stauchwallseite, die dem Eis zugewandt ist, Geschiebe nicht höher als $50 \mathrm{~m}$ über dem Meer liegen.

Die bisher gefundenen Geschiebe auf den niederrheinischen Stauchwällen von Moyland und Kranenburg liegen um 40-50 m über NN; auf den höheren Stauchwallpartien von Kleve-Kranenburg konnten bisher keine nordischen Erratica gefunden werden. Ähnliche Beobachtungen machte STEEgER (1931) im Raum Moers, der nordische Erratica auf dem 
Egelsberg in $47 \mathrm{~m}$ Höhe fand. Dies bedeutet, daß das Gletschereis nicht über die höheren Stauchwälle hinwegglitt, sondern in der dem Eis zugewandten Hanglage der Stauchwälle steckenblieb.

Die Stauchwälle von Kleve-Kranenburg sind nicht vom Eis überfahren worden; entsprechendes konnte Thоме (1958) für den Schaephuysener-Höhenzug nachweisen (Höhen zwischen 60 und $80 \mathrm{~m}$ über NN). De ZANGER (1980) bemerkt, daß der Stauchwall von Arnheim während der maximalen Ausbreitung des Gletschers nicht vom Eis überfahren, aber teilweise mit Eis bedeckt gewesen ist. Niedrige Stauchwälle - wie der von Moyland mit der höchsten Erhebung um 48 m (Abb. 2; Beilage 1 in SieberTz 1980) - sind vom Eis überflossen worden (vgl. Heine 1983; Siebertz 1980, 1983).

\section{Die Bedeutung der Stauchwälle von Kleve-Kranenburg für die glazialmorphologische Entwicklung des nördlichen Niederrheinischen Höhenzuges}

Nach Klostermann (1981) und MaArleveld (1981) lassen sich in den Stauchwällen von Kleve-Kranenburg morphologisch zwei Eisvorstöße nachweisen (vgl. Abb. 8). Dies geht nun auch klar aus den Grundmoränenvorkommen (I) und (II) im Aufschluß A 154 hervor (Abb. 7).

Die geomorphologischen Befunde im Raum Kleve lassen für die glazialmorphologische Entwicklung folgende Schlüsse zu: (1) Die Louisendorf-Staffel stieß auf breiter Front von ENE durch die heutige Rheinniederung bei Kalkar bis zu den Höhen von Louisendorf vor; mit ihr wurde der Vorschüttsand zwischen Kleve und Uedem abgelagert, dessen Schmelzwässer die ausgeprägten, parallel zu den Stauchwällen in die Niersniederung nach SW verlaufenden fluvioglazialen Rinnen hinterließen. Mit dem Abschmelzen des Eises wurde der Nachschüttsand sedimentiert, dessen Grenze sich von SE bis nach Kleve über den gesamten Höhenzug verfolgen läßt (Abb. 2).

Die Sandersedimente der Louisendorf-Staffel bilden aufgrund der fehlenden Endmoräne bzw. des Stauchwalls den Endmoränen-Vertreter im Sinne von GrIPP (1975). Auf der Louisendorfer-Höhe erreichte das Eis seine maximale Ausdehnung; diese wird durch die Grenze zwischen Vor- und Nachschüttsand dokumentiert (Abb. 2; Beilage 1 in Siebertz 1980).

Dem Eisvorstoß von Louisendorf (Louisendorf-Staffel) scheint somit eine größere Bedeutung zuzukommen, als dies von HeIne (1983) angenommen wurde, der ihn als kleine Besonderheit am Eisrand der Neußer-Staffel von Thоме (1959) betrachtete. Die Annahme von Siebertz (1980), daß die geringe Ausdehnung des Stauchwalls von Moyland auf seine Stellung zwischen den beiden Eisloben im NW von Kleve und im SE von Xanten abhängt, ist nur für den zweiten Eisvorstoß (Moyland-Staffel) zutreffend.

(2) Die Moyland-Staffel bildete den Stauchwall von Moyland und hinterließ keine nennenswerten Sandersedimente. Für diesen Eisvorstoß gibt es im Raum Kleve bisher keine Hinweise; entweder haben sie nie existiert, oder sie sind der Erosion zum Opfer gefallen. Grundmoräne zu diesem Eisvorstoß ist lediglich im Raum des Stauchwalls von Moyland bekannt (vgl. SieberTz 1983).

(3) Für die Stauchwälle von Kleve-Kranenburg sind zwei Gletschervorstöße verantwortlich (Abb. 2), die unabhängig von den Vorgängen im Raum Louisendorf-Moyland zu betrachten sind. Morphologisch werden sie durch zwei Stauchwallreihen (Klever-Staffel; 

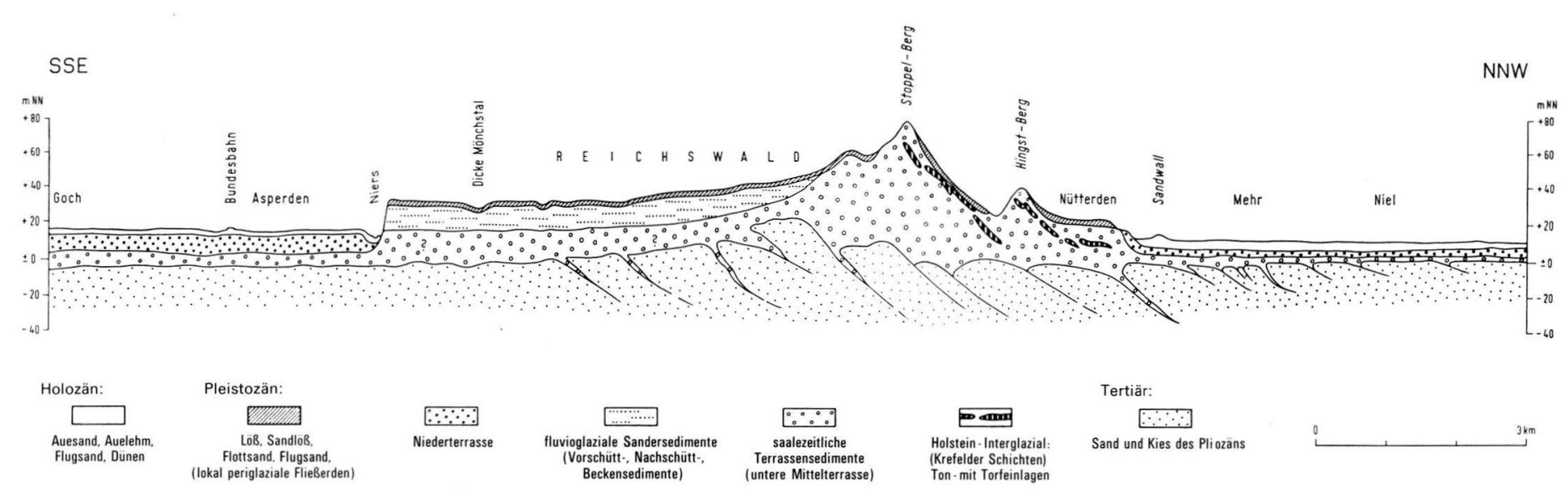

Abb. 8: Querprofil durch Sanderhochfläche und Stauchwälle bei Kleve-Kranenburg (verändert nach BRAUN 1968). 
Kranenburger-Staffel) und durch die Grundmoränen (I) und (II) belegt (Abb. 7). Die aus NNW vorrückenden Gletscher sind relativ jünger als die Gletschervorstöße von Louisendorf-Moyland; sie blieben in den bereits abgelagerten Sedimenten der LouisendorfStaffel stecken. Auf das glazialmorphologische Geschehen im südlichen Raum der Stauchwälle hatten sie keinen (oder geringen) Einfluß.

Die Abb. 8 täuscht aufgrund der Profillage durch die Stauchwallrücken bei Kleve und dem anschließenden Sander (Reichswald) eine morphologische Einheit und Abhängigkeit vor, die von der Genese her nicht vorhanden ist. Für alle Stauchwallrücken am Niederrhein (Xanten, Schaephuysen) läßt sich belegen, daß markante Stauchwälle und ausgedehnte Sanderflächen einander ausschließen.

\section{Zusammenfassende Erkenntnisse für die Entwicklung der Stauchwälle von Kleve-Kranenburg}

Der Raum Kleve-Kranenburg bildet mit seinen Stauchwällen, Grundmoränenvorkommen und Sanderablagerungen einen glazialmorphologischen Angelpunkt zwischen den aus zwei Richtungen vorrückenden Gletschern im Saale-Glazial am unteren Niederrhein. Folgende Befunde lassen sich zusammenfassen:

1. die Stauchwälle von Kleve-Kranenburg sind von zwei Gletschervorstößen gebildet worden; dies läßt sich nachweisen

a) morphologisch durch zwei Stauchwallreihen

ß) sedimentpetrographisch durch die Grundmoränen (I) und (II).

2. der Raum Kleve wurde von folgenden Eisvorstößen beeinflußt; es sind

a) die Louisendorf-Staffel aus ENE, deren Sander den Niederrheinischen Höhenzug zwischen Kleve und Uedem aufbaut. Sie wird dokumentiert durch die Grenze zwischen Vor- und Nachschüttsand, die sich über den gesamten Höhenzug verfolgen läßt. Die Moyland-Staffel läßt sich im Raum Kleve (bisher) nicht nachweisen.

ß) die Gletschervorstöße der Klever-Staffel und Kranenburger-Staffel, die im Vorschüttsand der Louisendorf-Staffel stecken blieben und zu dieser keine (unmittelbare) Beziehung aufweisen. Diese Gletschervorstöße müssen unabhängig voneinander betrachtet werden, wobei die Louisendorf-Staffel gegenüber der Klever-Staffel als relativ älter $\mathrm{zu}$ betrachten ist.

3. die Schmelzwässer der Gletscher von Kleve-Kranenburg haben am Sanderaufbau zwischen Kleve und Uedem keinen (direkten) Anteil; dies läßt sich nachweisen

a) durch die parallel zu den Stauchwällen von Kleve-Kranenburg verlaufenden Schmelzwasserrinnen der Louisendorf-Staffel, die zur Klever-Staffel keine Verbindung aufweisen.

ß) durch den Umstand, daß die Stauchwälle nicht vom Eis überflossen worden sind, was durch das Fehlen von Grundmoräne auf den Stauchwallhöhen bestätigt wird.

$\gamma$ ) durch die sedimentpetrographische und schwermineralogische Zusammensetzung des Sanders, welche die Abhängigkeit zur unteren Mittelterrasse erkennen läßt. Der Einfluß von nordischem Geschiebe auf das leicht- und schwermineralogische Bild des Sanders wird erst durch die Einarbeitung der Grundmoräne (Moyland-Staffel) in die Beckensedimente im Stauchwall von Moyland bestätigt. 


\section{Schriftenverzeichnis}

Braun, F. J. (1956): Die Terrassengliederung am linken Niederrhein zwischen Geldern und Kalkar. - Geologie en Mijnbouw (N.S.), 18: 374-378, 3 Abb.; s'Gravenhage.

- (1959): Endmoränen, Terrassen und holozäne Ablagerungen bei Xanten am Niederrhein. Fortschr. Geol. Rhld. u. Westf., 4: 247-254, 2 Abb., 1 Tab., 1 Taf.; Krefeld.

- (1964): Endmoränen-Stauchwall und Eisrandbildungen bei Moyland/Ndrh. - Der Niederrhein, 2: 58-63, 7 Abb., 1 Tab.; Krefeld.

- (1968): Übersichtskarte von Nordrhein-Westfalen 1:100.000, Erläuterungen zur geologischen Karte C 4302 Bocholt, 13-92, 12 Abb., 4 Tab., 4 Taf.; Krefeld (Geol. L.-Amt NordrheinWestfalen).

- (1978a): Geschiebekundliche und mineralogisch-petrologische Besonderheiten im EndmoränenStauchwall von Moyland bei Kalkar/Ndrh. - Fortschr. Geol. Rhld. u. Westf., 28: 325-333, 2 Abb., 1 Tab., 1 Taf.; Krefeld.

- (1978b): Zur Herkunft und Zusammensetzung des "Sandlösses« auf der Uedemer SanderHochfläche (Niederrhein). - Fortschr. Geol. Rhld. u. Westf., 28: 335-343, 5 Abb., 3 Tab.; Krefeld.

De Jong, J. D. (1956): Sedimentpetrographische Untersuchungen in Terrassenschottern im Gebiet zwischen Krefeld und Kleve. - Geologie en Mijnbouw (N.S.), 18: 389-394, 5 Abb., 1 Tab.; s'Gravenhage.

De ZANGER, F.A.P. (1980): Die Höhenlage des Eises des Gletscherlobus während der Formung des Stauchwalls von Arnheim (Niederlande). - Eiszeitalter u. Gegenwart, 30: 19-28, 4 Abb., 1 Tab.; Hannover.

Edelman, C.H. \& MaArleveld, G.C. (1958): Pleistozän-geologische Ergebnisse der Bodenkartierung in den Niederlanden. - Geol. Jb., 73: 639-684, 26 Abb., 3 Tab., 2 Taf.; Hannover.

GRIPP, K. (1974): Untermoräne-Grundmoräne-Grundmoränenlandschaft. - Eiszeitalter u. Gegenwart, 25: 5-9, 1 Abb.; Öhringen.

- (1975): Hochsander-Satzmoräne-Endmoränenvertreter. - Z. Geomorph. N.F., 19: 490-496, 3 Fig., 1 Phot.; Berlin-Stuttgart.

HeINE, K. (1983): Der Vorstoß des nordeuropäischen Inlandeises am Niederrhein (Raum KleveKalkar-Goch). - Beiträge zum Quartär der nördlichen Rheinlande, Arb. z. Rhein. Landeskde, 51: 39-49, 4 Abb.; Bonn.

- \& Siebertz, H. (1980): Abriß der paläogeographischen Entwicklung des unteren Niederrheingebietes. - Niederrheinische Studien, Arb. z. Rhein. Landeskde, 46: 1-13, 6 Abb., 1 Tab.; Bonn.

Henningsen, D. (1983): Der Schwermineral-Gehalt der Drenthe-zeitlichen Schmelzwassersande in Niedersachsen. - Eiszeitalter u. Gegenwart, 33: 133-140, 3 Abb.; Hannover.

KAISER, K.H. (1961): Gliederung und Formenschatz des Pliozäns und Quartärs am Mittel- und Niederrhein, sowie in den angrenzenden Niederlanden unter besonderer Berücksichtigung der Rheinterrassen. - Kayser, K. u. Kraus, Th. (Hrsg.), Köln und die Rheinlande, Festschr. XXXIII Deutschen Geographentag, 236-278, 6 Abb., 7 Tab., 3 Kart.; Wiesbaden.

- \& SchütrumpF, R. (1960): Zur Gliederung mittel- und jungpleistozäner Schichten in der Niederrheinischen Bucht. - Eiszeitalter u. Gegenwart, 11: 166-185, 5 Abb., 2 Tab.; Öhringen.

KlostermanN, J. (1981): Das Quartär der nördlichen Niederrheinischen Bucht. - Der Niederrhein, 48: 79-85, 150-153, 212-217, 10 Abb., 1 Tab.; Krefeld.

MaArleveld, G.C. (1956): Ergebnisse von Kies-Analysen im Niederrheingebiet. - Geologie en Mijnbouw (N.S.), 18: 411-415, 1 Abb., 2 Tab.; s'Gravenhage.

- (1981): The Sequence of Ice-Pushing in the Central Netherlands. - Meded. Rijks Geol. Dienst, 34: 2-6, 2 Fig.; Den-Hag. 
SIEBERTZ, H. (1980): Ausgewählte quartärmorphologische Probleme am unteren Niederrhein. Ergebnisse einer geomorphologischen Kartierung, dargestellt am Beispiel einer geomorphologischen Übersichtskarte vom Raum Kalkar. - Niederrheinische Studien, Arb. z. Rhein. Landeskde, 46: 37-46, 3 Abb., 2 Kart.; Bonn.

- (1983): Sedimentologische Zuordnung saalezeitlicher Gletscherablagerungen zu mehreren Vorstößen am unteren Niederrhein. - Eiszeitalter u. Gegenwart, 33: 119-132, 6 Abb., 2 Tab.; Hannover.

Steeger, A. (1931): Die Terrassen- und Endmoränenlandschaft zwischen Krefeld, Moers und Geldern. - Die Natur am Niederrhein, 7: 6-22, 17 Abb.; Krefeld.

Thiermann, A. (1970a): Geologische Karte Nordrhein-Westfalen 1:25.000, Erl. Bl. 3712 Tecklenburg: 243 S., 22 Abb., 10 Tab., 7 Taf.; Krefeld (Geol. L.-Amt Nordrhein-Westfalen).

- (1970b): Geologische Karte Nordrhein-Westfalen 1:25.000, Erl. Bl. 3711 Bevergern: 120 S., 9 Abb., 6 Tab., 5 Taf.; Krefeld (Geol. L.-Amt Nordrhein-Westfalen).

Tноме, K.N. (1958): Die Begegnung des nordischen Inlandeises mit dem Rhein.-Geol. Jb., 76: 261-308, 11 Abb.; Hannover.

- (1959): Eisvorstoß und Flußregime an Niederrhein und Zuider See im Jungpleistozän. - Fortschr. Geol. Rhld. u. Westf., 4: 197-246, 19 Abb., 5 Tab., 1 Taf.; Krefeld.

VAN ANDEL, T.H. (1952): Zur Frage der Schwermineralverwitterung in Sedimenten. - Erdöl u. Kohle, 5: 100-104, 2 Abb., 2 Taf.; Hamburg.

W AGNER, E. (1968): Das niederrheinische Tiefland. - Topogr. Atlas Nordrhein Westfalen, 208-209, 3 Abb.; Bad Godesberg.

WeYL, R. (1949): Zur Ausdeutbarkeit der Schwermineral-Vergesellschaftungen. - Erdöl u. Kohle, 2: 221-224, 3 Abb., 3 Zahlentaf.; Hamburg.

- (1950): Schwermineralverwitterung und ihr Einfluß auf die Mineralführung klastischer Sedimente. - Erdöl u. Kohle, 3: 209-211, 7 Abb.; Hamburg.

ZaNDSTRA, J.G. (1976): Sedimentpetrographische Untersuchungen des Geschiebelehms von Emmerschans (Drenthe, Niederlande) mit Bemerkungen über eine Typeneinteilung der SaaleGrundmoräne. - Eiszeitalter u. Gegenwart, 27: 30-52, 7 Abb., 6 Tab.; Öhringen.

ZoNNEveld, J.I.S. (1956): Schwermineralgesellschaften in Niederrheinischen Terrassensedimenten. Geologie en Mijnbouw (N.S.), 18: 395-401, 2 Fig., 2 Tab.; s'Gravenhage.

Manuskript eingegangen am 5. 7. 1984, Nachträge September 1984. 